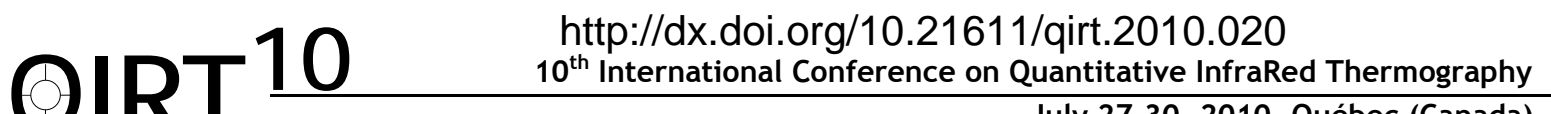 \\ July 27-30, 2010, Québec (Canada)
}

\section{Random photothermal thermography: Principle and examples of applications}

by Jean-Luc Bodnar, Jean-Charles Candoré, Jean-Louis Nicolas et Didier Caron

\author{
GRESPI/ECATHERM, UFR Sciences Exactes et Naturelles, BP 1039, 51687 Reims cedex 02 \\ (Corresponding author: jl.bodnar@univ-reims.fr)
}

\begin{abstract}
The photothermal thermography is a procedure of non-destructive control which finds numerous applications in the field of the control and characterization of the thin materials. This technique is classically implemented under CW excitation or under flash analysis (crenel). In our laboratory, we looked, just like what we had made in punctual photothermal radiometry, to implement it under random excitation. This last technique seems to allow indeed, with regard to the previous ones, the control of more fragile materials as well as the access to extend multi frequencies analysis. Having reminded the principle of the random photothermal thermography and having presented an example of experimental device, we show that the method allows under lesser energy constraints, on one hand the detection of detachments situated in mural paintings and on the other hand, detection of a defect situated in a block of plaster while leading only a weak rise of temperature.
\end{abstract}

\section{Introduction}

The problem of the non-destructive testing of materials is for a long time a subject which interests the industrialists. We can measure the importance of this problem among studies carried out and for the variety of the proposed techniques. We can quote the $\mathrm{X}$-rays and the $\gamma$-rays radiography, the ultrasounds, the Eddy currents, the penetrant testing, the magnetoscopy and the optical techniques.

The infrared photothermal thermography is one of these last optical techniques. Its principle consists in subjecting the sample to be analyzed in a flow of photons which absorption produces a local rise of temperature in the vicinity of the light impact point, then to observe the variations of emissions of the material by means of an infrared camera of thermography.

The thermophysical phenomena mainly implemented by this check procedure are the conduction and the thermal radiation. The photothermal signal collected by the infrared radiometer depends on parameters governing these physical phenomena (thermal conductivity, thermal diffusivity ...), but also on all the parameters being able to be correlated to these last ones (presence of delamination, presence of crack ...). The method offers to the engineers a means of characterization of these various parameters [1-7].

The principle of this method makes it is non-destructive, without contact, flexible, easily customizable according to needs and finally it allows the study of the first 100 micrometers of materials (what is not still possible with the others procedures of non-destructive testing). It also makes the method is limited to the study of the thin materials (up to some centimeters in thickness) and it can be dependent on radiative properties of surface.

Until now, to check samples by infrared photothermal thermography, two modes of analyses are rather implemented (because of their well known capacities); the one, under CW excitation and Lock - in detection, the other one under pulsed excitation and continuous detection. In the first case, the method indeed allows, to obtain a very good ratio signal to noise as well as the access to two characteristic parameters: the magnitude and the phase of the photothermal signal. In the second case, the method allows to obtain a response very rich in information, because giving access to the pulse response of the material.

These two methods present however inconveniences which limit their field of application. In the case of the CW excitation, it is essentially about the slowness of analysis which allows with difficulty an industrial analysis. In the second case, it is about the obligation, to obtain an excitation close to a Dirac function, to deposit an important energy during a short lapse of time what does not authorize the analysis of fragile materials (biological materials, works of art...). To extend the field of investigation of the photothermal thermography, new studies and new modes of analysis are to be developed again.

For some years, thanks to the computerization more and more pushed of the instrumentations and to the development of the methods of signal processing, appear in numerous domains of physics, new methods of analysis, complementary of those traditionally used (Fourier and flash analysis). One of them consists in associating a random excitation and a correlation or a parametric analysis with the mode of used control. The main advantage of this association, 
with regard to the classic techniques, is then to give access to the pulse and harmonic multi frequencies responses of the materials, while implementing a lesser density of excitation.

The idea to join a random excitation and a correlation or a parametric analysis to the infrared thermography is then attractive, because it could offer new possibilities in non-destructive control. That is why, for several years, we were interested in this association.

First, we implemented a localized excitation and a punctual detection (SAMMIR system) and showed the random method allowed, under lesser energy constraints, to make actions of non-destructive control and thermal metrology [8-9]. However, the instrument used for these studies was rather an instrument of laboratory, with difficult use on site. As eventually, we wished develop actions of control «in situ» and it, in an industrial way, we tried to develop a new tool of photothermal analysis, portable, allowing a extend analysis, and especially under thermal constraint the weakest possible. It is the new tool of non-destructive control we realized with this objective, we present here.

We present at first the principle of a random photothermal analysis.

We present then the advantages of this checking procedure.

We present then the device of random photothermal thermography of the laboratory: the SAMMTHIR system.

We show finally the method allows on one hand, the non-destructive analysis under lesser energy constraints of mural paintings, in laboratory and in situ and on the other hand the detection of a defect situated in a block of plaster while leading only a weak rise of temperature.

\section{Principle of random photothermal analysis}

The principle of the random photothermal thermography consists in exciting the material to study by means of a signal approaching a white noise, then to reconstruct the pulse response and/or the harmonic response of the sample by a correlation analysis (statistical analysis), or by a parametric analysis (study of a model of behavior) of the photothermal response obtained. The first type of analysis, the correlation analysis, consists in calculating the pulse response of the material studied from the product of inter-correlation between the photothermal response of the studied system $s(t)$ and the sequence of excitation e(t) used. The harmonic photothermal multi frequencies response of the material is then calculated by Fourier transforms of this pulse response (Fig. 1.).

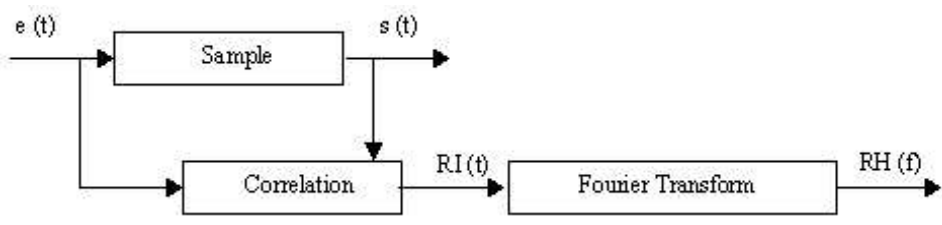

Fig. 1. Principle of a correlation analysis

The principle of the second mode of analysis, the parametric analysis, consists in reconstructing in theory the pulse and harmonic responses of the studied physical system, from a behavior model, mostly the type ARMA, of this last one; this model being built from the analysis of the photothermal response of the sample studied in front of an excitation the whitest possible (Fig.2.)

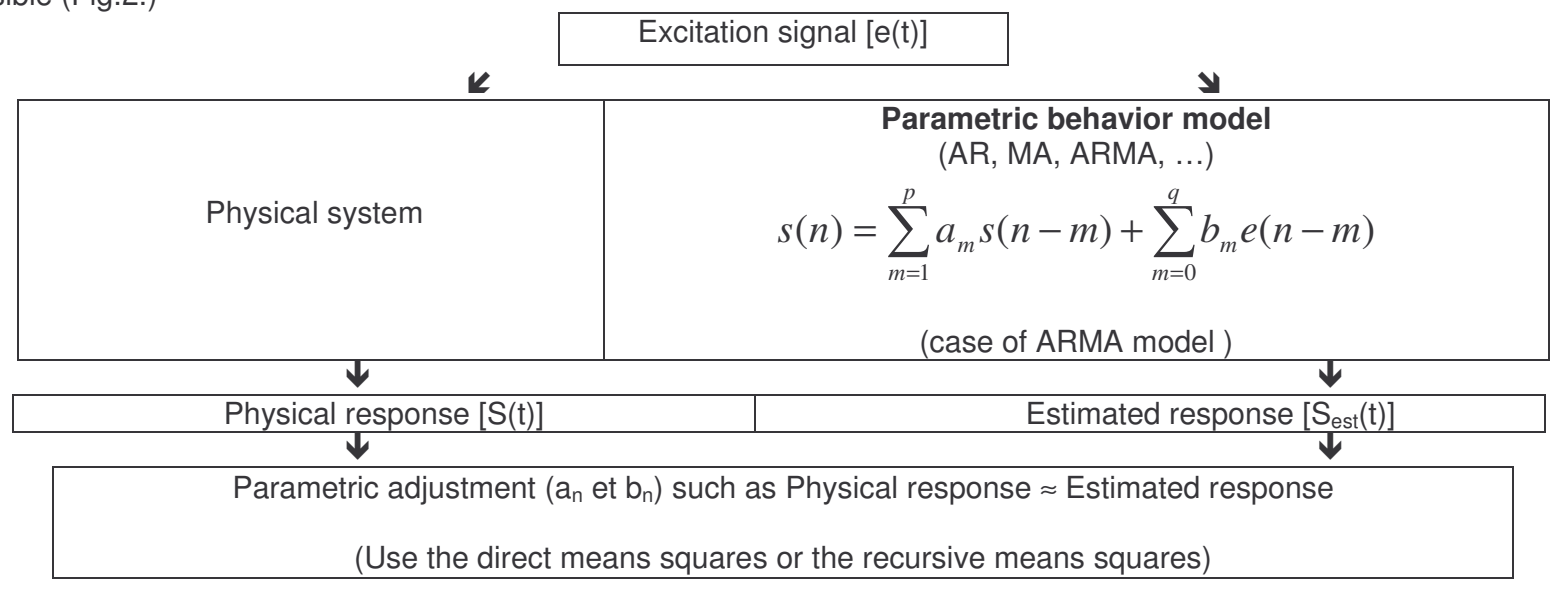

Fig.2. Principle of construction of a behavior model by parametric analysis 
The construction of the pulse response is made by calculating the response of the behavior model in front of a Dirac function. The construction of the harmonic response is made by calculating Fourier transform of this pulse response (Fig. 3.).

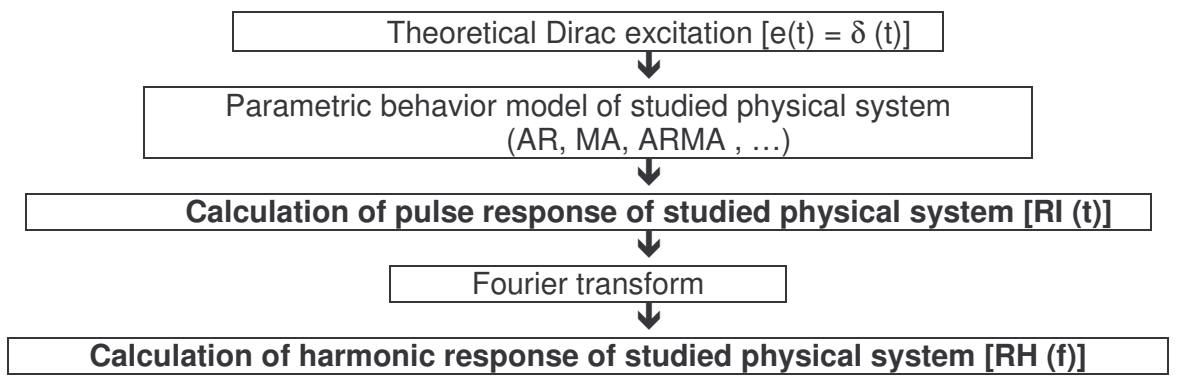

Fig. 3. Principle of parametric identification of physical system

Finally to build the white noise necessary for these two modes of analyses, for the first case, to simplify the calculation of correlation and in the second case to build a behavior model, the finest possible, we used the procedures proposed by J.Auvray [10]. This last one suggests three different manners approaching this theoretical signal: either build a pseudo random binary sequence, or build a Gaussian signal or build a signal with frequency scanning.

In our studies, essentially for reasons of ease of implementation, it is rather the pseudo random binary sequences we implemented. In that case, the mode of construction of the pseudo random binary sequences consists then, to collect the signal delivered at the exit of a register composed of $D$ flip flop, locked up on itself via a modulo 2 addition (Fig. 4.); the exits of the $\mathrm{D}$ flip flop taken into account during this addition were the ones defined by the primitives polynomials associated with the procedure of creation (Fig. 5.). An example of pseudo random binary signal is presented on the Fig. 6 .

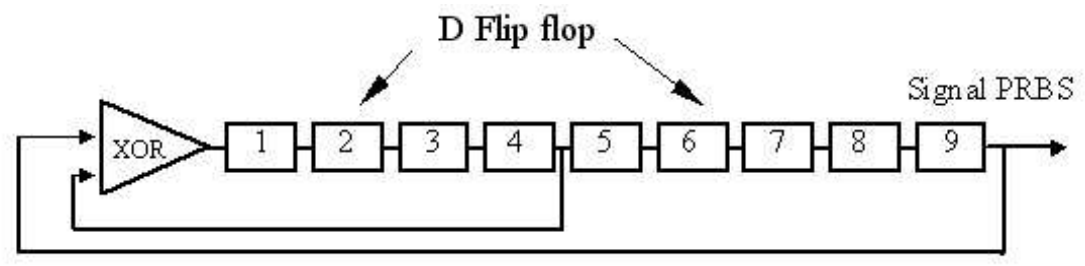

Fig. 4. Principle of creation of pseudo random binary sequence

\begin{tabular}{|c|c|}
\hline degrees & Primitives polynomials \\
\hline & \\
\hline 2 & $X^{2}+X+1$ \\
\hline 3 & $X^{3}+X^{2}+1$ \\
\hline 4 & $X^{4}+X+1$ \\
\hline 5 & $X^{5}+X^{2}+1$ \\
\hline 6 & $X^{6}+X+1$ \\
\hline 7 & $X^{1}+X^{3}+1$ \\
\hline 8 & $X^{6}+X^{4}+X^{3}+X^{2}+1$ \\
\hline 9 & $X^{9}+X^{4}+1$ \\
\hline 10 & $X^{10}+X^{3}+1$ \\
\hline
\end{tabular}

Fig. 5. Table of primitive polynomials used to generate pseudo random binary sequence 


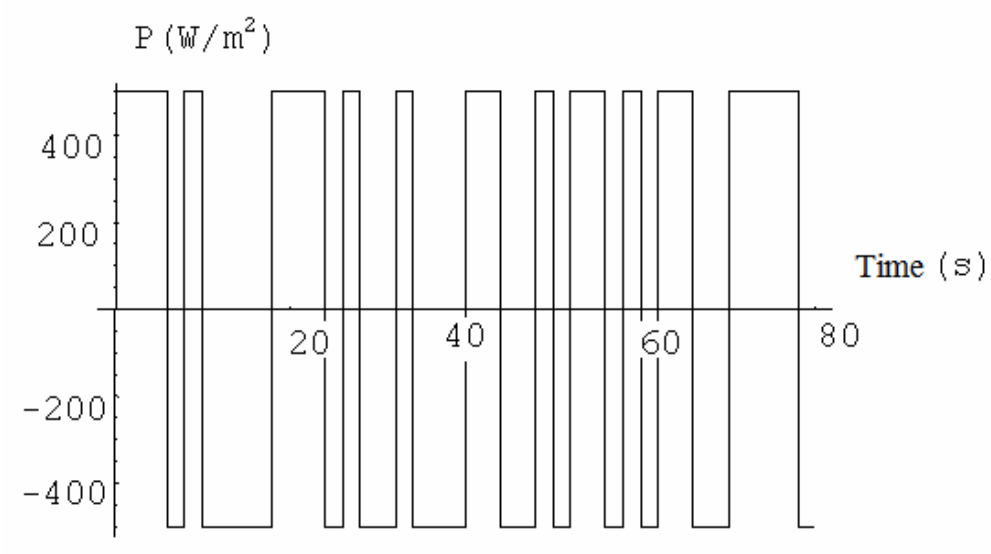

Fig. 6. Example of excitation signal usable for the analysis: a pseudo random binary signal

\section{Potential applications of random photothermal thermography}

As we saw it, the random photothermal thermography has for main advantages to allow non-destructive analyses, made under lesser energy constraints and to give access to three types of photothermal signals: the raw random response (analyzable by direct deconvolution), the pulse response and the harmonic response of the analyzed material (analyzable by the analysis tools developed for flash and harmonic analyzes). These specificities make the method seems to be adapted well to the following applications:

* The non-destructive control of fragile samples such as works of art, biological materials, wall painting of houses or covered with wallpaper ...

* The tomographic control of sample via multi frequencies or temporal analyzes.

* The precise measure, because of the lesser energy disturbance engendered by the instrument of measure, of thermophysical properties, via the analysis of one of three types of signals supplied by the method.

* The in situ characterization of materials or industrial processes (the random analysis can be made in addition of an already existing request).

Besides, the low density of power deposited during the photothermal analysis makes the instrument requires only a weak power supply (a simple classic socket 220 volts / $16 \mathrm{~A}$ is enough) to collect the photothermal response of the material analyzed (while for example, a flash analysis asks mostly a more complex device of power supply in energy). It is then potentially very portable.

Finally, the fact of being able to modulate power, the deposited bright power, made the random method also allows to modulate the spectral range in which is deposited the excitation (by supply in power more or less the excitation lamps) and thus try to working in an absorption spectral range of the analyzed material.

\section{Presentation of a random photothermal thermography system: the SAMMTHIR system of the laboratory}

The random photothermal thermography system we developed in the laboratory is the SAMMTHIR system. It is made up of an optic of excitation, an optic of detection and finally an electronic and a computing of piloting of the instrumentation. The source of excitation is a couple (so as to return the deposit of more homogeneous energy) of halogens lamps. This source of excitation is modulated in a pseudo random binary way by means of electronics and of a computing of piloting. The system of detection is constituted by a camera of infrared thermography working in a synchronous way with the system of random excitation. The reconstruction by correlation or parametric analysis of pulse and harmonic multi frequencies responses is made in a module of data processing. Finally, a module of analyze of theses different responses completes the system (Fig.7.). 


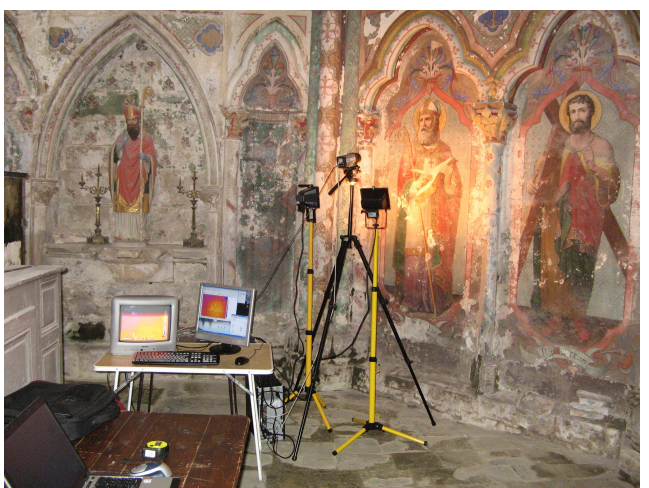

Fig. 7. Experimental device implemented for the study

\section{Examples of experimental results obtained}

The first example of result we present here, concerns the study of a mural painting that is a potentially fragile sample (one of the targets privileged by the random variant of the photothermal thermography). This fresco was realized according to the technique of primitive Italian by Gabriela Szatanik, professional restorer. It contains 4 inclusions of "plastazote" (Fig. 8.). The objective of the study was to verify that the random photothermal thermography allowed developing actions of non-destructive control and thus the detection of these defects. The experimental conditions selected are a pseudo random binary type excitation of 256 terms, a frequency of sampling of $0,1 \mathrm{bit} / \mathrm{s}$, a model of behavior of type ARMA, a number of entry and exit parameters of this model equals to 40 . An example of result obtained is presented on the Fig. 8 . On this figure, we drew, in coding false colors, for every pixel of the image, the area contained under the pulse photothermal response reconstructed by parametric analysis (so as to group together on the same synthetic image all the information given by the pulse response) This figure, because of the effect of thermal barrier worked by the defects, makes clearly appear an infrared signature more important for their place. It confirms thus the random photothermal thermography can be a new tool of non-destructive control.

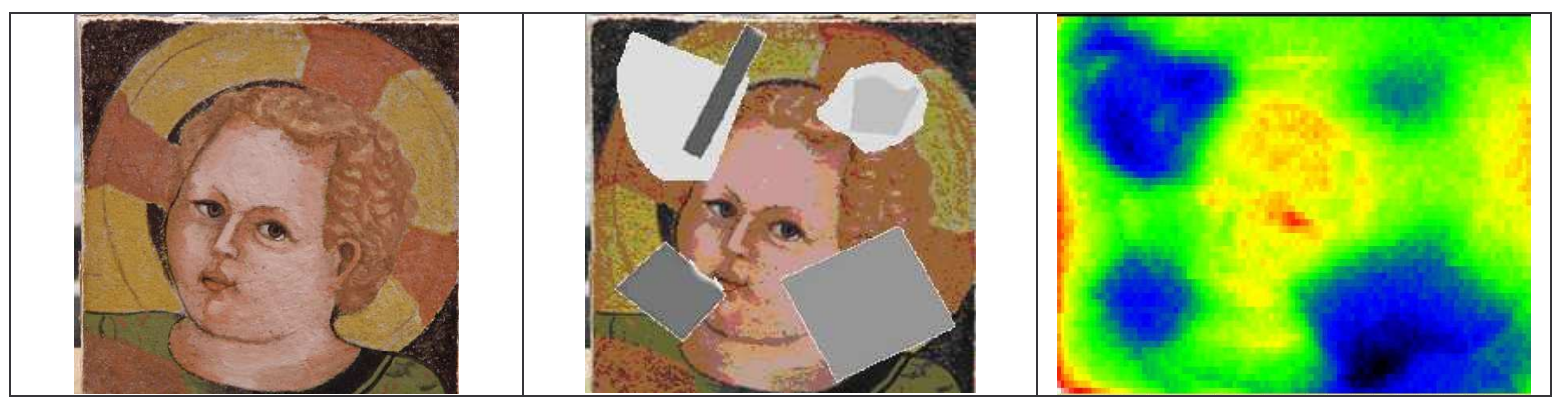

Fig. 8. The fresco studied (left), the location of internal defects (center) and an example of random photothermal results obtained (right)

Following this first analysis, rather positive, but developed in laboratory, we are having developed an in situ analysis of work of art. The mural painting we analyzed is a painting on canvas, dating the 19th century, and placed in the apse of the church of Bonnet. It represents "Saint Martin" (Fig. 9.). 


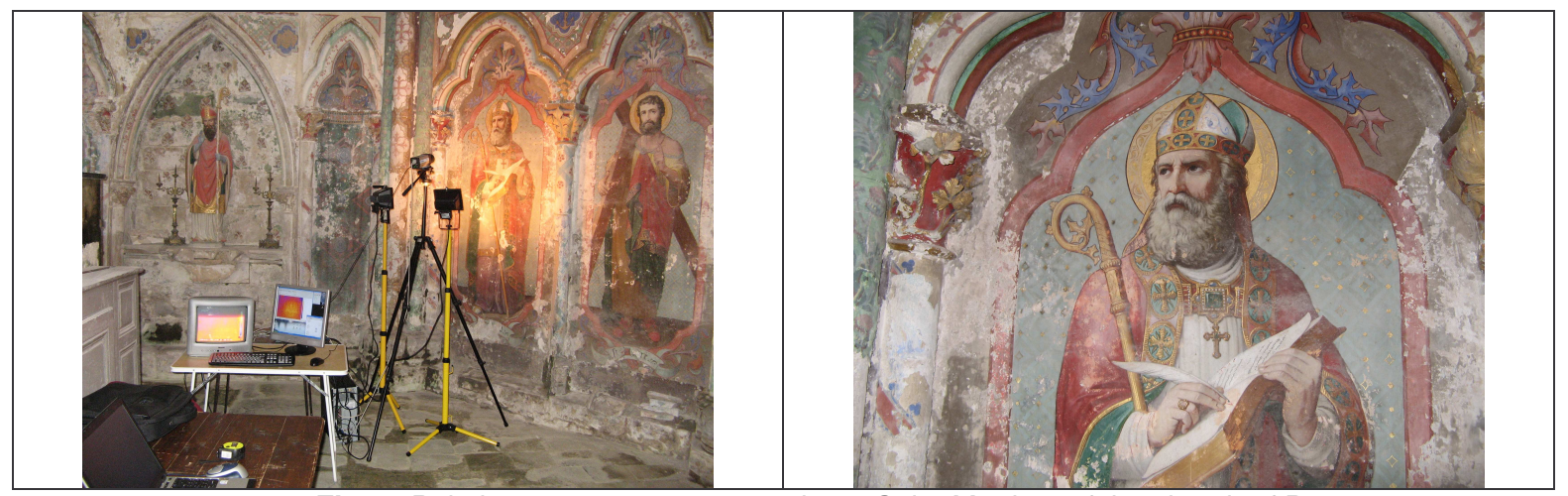

Fig. 9. Painting on canvas representing " Saint Martin » of the church of Bonnet

We analyzed first of all this painting on canvas in an acoustic way. The results obtained makes appear a multitude of "hollow" tones, distributed rather in periphery of the work of art (Fig. 10.).

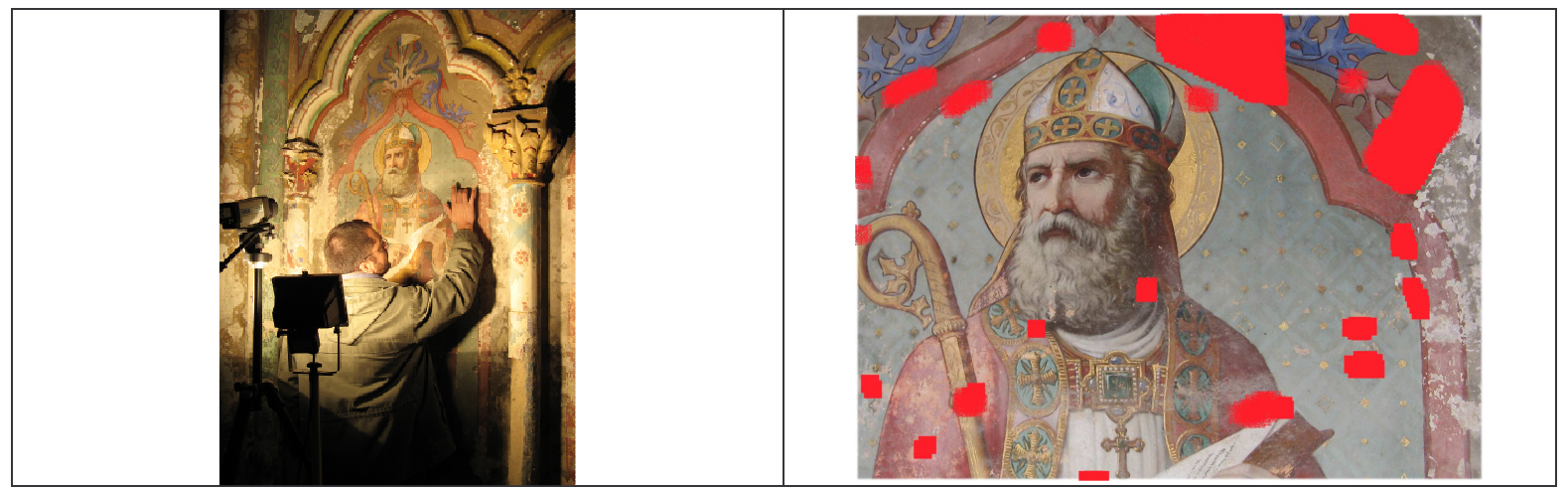

Fig. 10. Acoustic analysis of painting on canvas representing "Saint Martin » of the church of Bonnet

We then analyzed this painting canvas by stimulated infrared thermography, on one hand under pulsed excitation and continuous detection (excitation duration of 5 minutes), on the other hand under random excitation and parametric analysis (pseudo random binary excitation of 256 terms, ARMA model taking into account 40 parameters of entry and exit, analysis period equal to $15 \mathrm{~s}$ ).

The photothermal results obtained are presented on the Fig. 11., on left for the pulsed analysis and right for the random analysis. It is in the first case the infrared image obtained at the end of excitation. It is in the second case an image of the pulse response reconstructed by parametric analysis.

These two images show at first, more important photothermal signatures in places referenced by the acoustic analysis. They show consequently that the random thermography can be a new tool of in situ non-destructive testing method.

These two images show then, the image obtained by random analysis seems finer and more resolute, than those obtained by pulsed analysis. Indeed it seems to allow a better localization of the defects (including when they are very close some of the others: upper right red zone of thermograms).

These two images show finally, that the random method also seems to allow an easier localization of the defects because making appear, in a implicit way, the pictorial layer.

If these two observations can be generalized, the random infrared thermography, besides the fact of allowing the analysis of materials under lesser energy constraints and of giving access to three types of different responses, would present two other important advantages. 


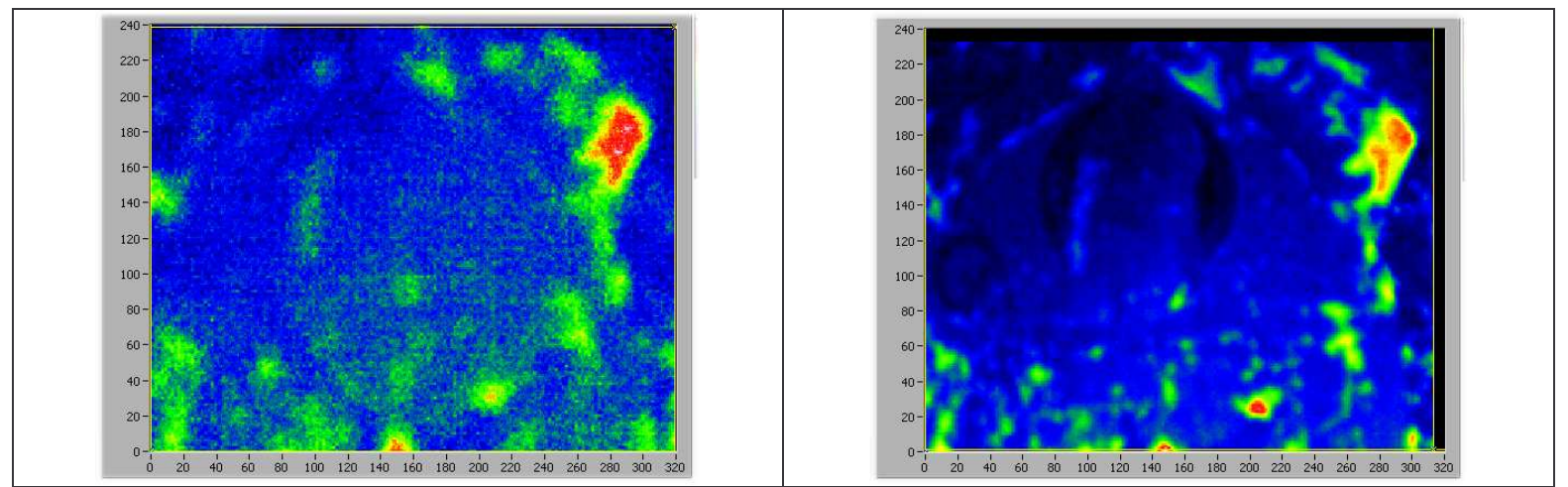

Fig.11. Pulsed and random photothermal analysis of painting on canvas representing "Saint Martin » of the church of Bonnet

The last example of result we present here concerns a quantitative approach of the thermal disturbance engendered by the random thermography analysis.

For that purpose, we studied a block of plaster $15 \mathrm{~cm}$ in length, $12 \mathrm{~cm}$ in width and 2,3 cm in thickness, containing a lack of mater $6,6 \mathrm{~cm}$ in length and $6,2 \mathrm{~cm}$ in width situated $8 \mathrm{~mm}$ under the central surface of the sample.

The experimental conditions retained for the study, are a pseudo random binary excitation of 128 terms, an electric power of $15 \mathrm{~W}$ (it is the lowest value allowed by our instrumentation), a period of excitation of $1 \mathrm{~s}$

An example of result obtained is presented on the Fig. 12. It represents the temporal evolution of the raw photothermal signature (what is sufficient here) of points situated on the surface of the studied sample, for the one situated directly above the defect and for the other one directly above the healthy part.

It makes appears, separate curves what confirms the possibilities of the random method in detection of defect.

It makes then appears a maximal rise of temperature of $1,56{ }^{\circ} \mathrm{C}$ what is relatively weak and in any case acceptable by the restorer to develop an analysis of mural painting.

It finally creates a maximal contrast of $0,33^{\circ} \mathrm{C}$, for a value equivalent to the noise of bolometric cameras of the market about $0,05^{\circ} \mathrm{C}$, what there also is acceptable.

To compare this result with the one that gives a classic flash analysis, we then studied the same sample by subjecting it to the shortest excitation and the most intense possible (Close to a Dirac function) which allows our instrumentation

We then excite the sample studied with an electric power of $1000 \mathrm{~W}$ during 1 second. An example of result obtained is presented on the Fig. 12. It represents also, the temporal evolution of the photothermal signature of points situated on the surface of the studied sample, for the one situated directly above the defect and for the other one directly above the healthy part. It shows that the rise of temperature engendered by the experiment leads a rise of temperature about $1,35^{\circ} \mathrm{C}$, that is a variation of the same order of height as in the previous case. But it also shows that the "flash" method in the conditions in the way we have implemented it does not simply allow the detection of the defect.

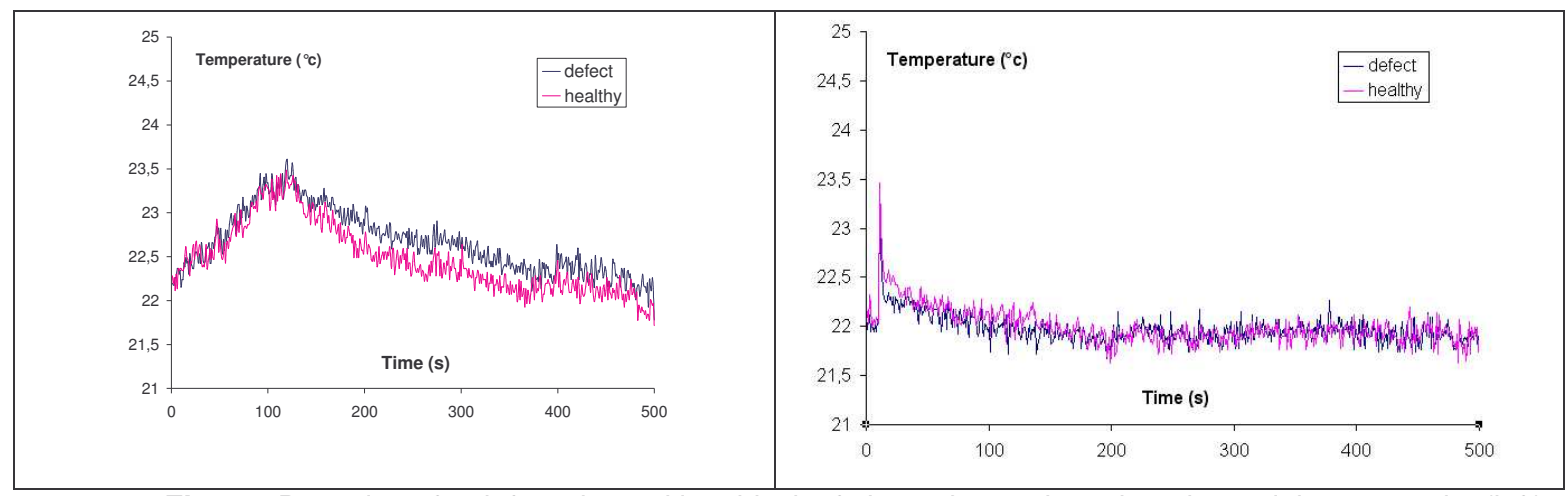

Fig. 12. Detection of a defect situated in a block of plaster by random photothermal thermography (left) and pulsed thermography (right) 


\section{Conclusion}

The recent progress of the optics, the electronics, the computation and of the techniques of signal processing leads to develop of news procedures of non-destructive testing. Among these, we proposed in this work, the random photothermal thermography.

We presented at first the principle of this method.

We then presented main advantages of this method.

In the third stage, we presented the device of random photothermal thermography developed in the laboratory (SAMMTHIR) and implemented for the study.

We finally showed that the method allows, on one hand the detection of defects of delamination situated in mural painting, it during studies developed in laboratory and during studies developed in situ. In this occasion, we underlined at first that the random method seemed to give access to finer and more resolute photothermal images, than those obtained by pulsed analysis. We also underlined that the random method seemed to allow an easier localization of the defects because making appear, in an implicit way, the motif of the pictorial layer.

We finally showed that the method allows, on the other hand the detection of a defect situated in a block of plaster while leading only a low rise of temperature.

These results obtained on particular samples have now to be generalized. Besides it would be also interesting to study the possibilities of the method in thermal metrology.

Studies going to this direction are in progress.

\section{REFERENCES}

[1] Maldague Xavier : Theory and practice of infrared technology for non destructive testing

[2] V.P. Vavilov : Non destructive testing handbook : thermal / infrared testing.

[3] J.L. Bodnar : le contrôle optique des matériaux par methods optiques infrarouges, livre Méthodes et techniques optiques pour l'industrie, collectif d'auteurs, ISBN 978-2-918241-00-3, nov 2009.

[4] Jean Charles Candoré, Gabriela Szatanik, J.L Bodnar, Vincent Detalle, Philippe Grossel : Infra-red photothermal thermography: A tool of assistance for the restoration of murals paintings? QIRT2006, Padoue, Italie

[5] Jean Charles Candoré, J.L Bodnar, Vincent Detalle, Philippe Grossel : Non destructive testing of works of art by stimulated infrared thermography, procceding de qirt 2008, Cracovie, Pologne

[6] Jean Charles Candoré, J.L Bodnar, Françoise Depasse, Vincent Detalle, Philippe Grossel : Approach of the characterization of delamination in mural paintings, procceding de qirt 2008, 2-3 juillet 2008, Cracovie, Pologne

[7] Jean Charles Candoré, Jean Luc Bodnar, Antoine Szeflinski et Laurent Ibos : Helps with the thermal diagnosis of the building : Detection of defects of insulation by stimulated infra-red thermography procceding de qirt 2008, 2-3 juillet 2008, Cracovie, Pologne

[8] J.L Bodnar, J.L. Battaglia : Thermophysical parameters estimation using the system identification approach. European workshops METTI 2005, 17-21 jan 2005.

[9] S. Brahim, J.L. Bodnar et P. Grossel : Thermal diffusivity measurement by photothermal radiometry under random excitation and parametric analysis, Proceeding of 15th International Conference on Photoacoustic and Photothermal Phenomena, Leuven, 19-23 July 2009

[10] J. Auvray Identification de processus. Techniques de l'ingénieur 1994 fiche r 306. 